\title{
Magnetic Resonance Elastography for Liver Fibrosis in Methotrexate Treatment
}

\author{
Deana D. Hoganson ${ }^{1}$, Jun Chen ${ }^{2}$, Richard L. Ehman ${ }^{2}$, Jayant A. Talwalkar ${ }^{3}$, Clement J. Michet Jr. ${ }^{1}$, \\ Meng Yin ${ }^{2}$, Cynthia S. Crowson ${ }^{4}$ Eric L. Matteson ${ }^{1,4^{*}}$ \\ ${ }^{1}$ Division of Rheumatology, Mayo Clinic, Rochester, USA; ${ }^{2}$ Department of Radiology, Mayo Clinic, Rochester, USA; ${ }^{3}$ Department \\ of Gastroenterology and Hepatology, Mayo Clinic, Rochester, USA; ${ }^{4}$ Department of Health Sciences Research, Mayo Clinic, Roch- \\ ester, USA. \\ Email: hogansond@gmail.com, \{chen.jun, ehman.richard, talwalkar.jayant, michet.clement, yin.meng, crowson, \\ "matteson.eric\}@mayo.edu
}

Received December $24^{\text {th }}, 2011$; revised February $2^{\text {nd }}, 2012$; accepted February $7^{\text {th }}, 2012$

\begin{abstract}
Introduction: Hepatic magnetic resonance elastography (MRE) allows for noninvasive assessment of liver fibrosis. The purpose of this study was to evaluate the usefulness of MRE in detecting and quantifying liver fibrosis in patients with rheumatoid arthritis (RA) who have received methotrexate (MTX). Methods: The association between mean liver stiffness value as determined by MRE and variables of interest was determined. The decision for a liver biopsy in participants with an abnormal liver stiffness was made based on clinical judgment. Results: Sixty-five RA patients were enrolled. Mean liver stiffness value by MRE was abnormal in 7 patients, suggestive of hepatic injury. As a result of findings from the MRE, biopsies were performed in 5 patients and all correlated with elevated liver stiffness values. Elevated mean liver stiffness values were associated with body mass index (BMI) $\left(\mathrm{OR}=1.18\right.$ per $1 \mathrm{~kg} / \mathrm{m}^{2} ; 95 \%$ CI: $1.03,1.36 ; p=0.017)$. Neither the total MTX dose nor the duration of MTX treatment was associated with mean liver stiffness value ( $p=0.51$ and $P=0.20$, respectively). Conclusion: MRE provides a reliable, non-invasive assessment of liver fibrosis in patients with RA receiving MTX. Patients with RA receiving MTX who have an elevated BMI may be at increased risk for chronic hepatic injury, regardless of MTX cumulative dose or duration of treatment.
\end{abstract}

Keywords: Methotrexate; Hepatic Magnetic Resonance Elastography; Liver Fibrosis; Rheumatoid Arthritis

\section{Introduction}

Methotrexate (MTX) has become the most frequently prescribed disease modifying antirheumatic agent (DMARD) for rheumatoid arthritis (RA) because of its efficacy, low cost and safety [1]. Despite its generally good tolerability, hepatotoxicity has remained an ongoing concern with MTX treatment, prompting development of guidelines for laboratory monitoring of liver tests by the American College of Rheumatology (ACR) in 1994 [2].

Aggregate data from several studies reported in the guideline recommendations showed that 103/719 (14\%) and 6/719 (0.8\%) of RA patients receiving MTX had mild (grade IIIA) and moderate (grade IIIB) fibrosis on liver biopsy findings, respectively [2]. Of these, 2/719 $(0.3 \%)$ had grade IV cirrhosis. Most of these patients were treated on average for less than 5 years.

Kremer et al. reported that when $\leq 49 \%$ of measured aspartate aminotransferase (AST) values were abnormal, the specificity of normal (Roenigk) biopsy grade was

"Corresponding author.
$97 \%$. However, a high frequency ( $\geq 50 \%$ ) of abnormal AST values was shown to be a poor predictor of abnormal liver histology with a sensitivity of $12 \%$ [3].

There is considerable variability in the frequency of abnormal liver tests in RA patients receiving MTX who are monitored per guideline recommendations [4,5]. Overall, abnormal values are most often transient and discontinuation of MTX for this reason was rare, with a discontinuation rate of only 95 patients/10,000 person-years [4]. Transient serum liver enzyme abnormalities may be attributed to dose adjustments in response to abnormal values [6], though Yaziki et al. showed this to be uncommon [5].

Early studies of MTX-related hepatotoxicity often attributed liver fibrosis to MTX without full consideration of confounding factors, particularly with respect to nonalcoholic steatohepatitis (NASH), a condition now more widely recognized [7]. Because of the apparently low rate of clinically significant, MTX-related hepatotoxicity, some have advocated less frequent monitoring of liver tests, particularly in the absence of risk factors for liver 
disease.

There is also uncertainty about whether the risk for liver fibrosis is increased with cumulative doses of MTX. Some studies have described a dose related effect of MTX on development of liver fibrosis [8,9], while others have not $[10,11]$. Overall, few studies have investigated the histologic effects of MTX on hepatotoxicity beyond 5 years of use [12]. In the population of today's RA patients, it is not uncommon for RA patients to be treated with MTX for periods well in excess of 5 years [13].

The study of MTX induced liver fibrosis is limited by the invasiveness of the gold standard for its detection, a liver biopsy. For this reason, the development of reliable noninvasive techniques to detect liver fibrosis has been an area of current interest and research. Hepatic magnetic resonance elastography (MRE) is an imaging technique that has recently been developed and allows for noninvasive assessment of liver fibrosis [14-18]. This technique exploits the physical characteristics of liver tissue to assess for organ abnormalities. With fibrosis, the liver becomes stiffer. The examination is performed in an MRI scanner. A speaker-like device is placed against the body wall over the liver to generate low-frequency mechanical waves. The propagating waves are imaged in the liver using a special MRI technique. The examination typically requires about one minute of imaging time. The information is automatically processed by the scanner to generate cross-sectional images called elastograms that quantitatively depict the stiffness of tissue. Previous studies have shown that MRE has a sensitivity and specificity for detecting all grades of liver fibrosis as compared to normal liver of $85 \%$ - 98\% and $91 \%$ - 99\%, respectively $[15,18]$. Hepatic steatosis does not influence this assessment $[15,18]$.

The goal of this study was to develop an initial assessment of the utility of MRE in investigating the presence and determinants of MTX induced liver fibrosis in patients with RA.

\section{Materials and Methods}

This study was approved by the Mayo Clinic Institutional Review Board. All subjects gave written informed consent prior to participation in the study. The study design is a case series of patients with RA who fulfilled the entry criteria, as specified below, and who underwent MRE as described below.

\subsection{Patients}

Patients with RA as determined by a board-certified rheumatologist receiving treatment with MTX for $>5$ years or who had at least one abnormal AST value in the two years preceding the MR scan (2007-2008) were eligible and recruited to participate in the study. Recruit- ment was accomplished by a combination of subject identification in the outpatient clinic and by recruitment letters mailed to patients receiving laboratory monitoring for MTX at our institution. Patients with established causes of chronic liver disease including hepatitis B or C, hematochromatosis, alpha-antitrypsin deficiency, history of alcoholism or alcohol use while taking MTX, treatment with medications which might cause fibrosis, as well as patients with contraindications to MR examination (hardware, claustrophobia), and pregnant women were excluded. Initial analysis after 53 patients completed an MRE scan shows a possible relationship between elevated BMI and liver stiffness. An additional 12 patients were then recruited with a BMI of greater than 30 regardless of duration of methotrexate treatment or liver test abnormalities. One patient who consented for the study was not able to complete the scan due to claustrophobia.

\subsection{Data Collection}

Relevant clinical data were abstracted from the inpatient and outpatient clinical record. Variables of interest included age, sex, body mass index (BMI), presence of rheumatoid factor and/or CCP antibody, duration of RA, total cumulative MTX dose, duration of MTX treatment, frequency of AST abnormalities while receiving MTX, frequency of AST abnormalities in the 2 years preceding MR scan, presence/treatment of hyperlipidemia, diabetes mellitus (DM) (specifically diagnosed and recorded in the medical record and/or receiving insulin or hyperglycemic agents), impaired fasting blood glucose (IFG) (100 - $125 \mathrm{mg} / \mathrm{dL}$ and/or specifically diagnosed and recorded in the medical record), folate supplementation, and alcohol intake (none, one drink monthly, 2 - 4 drinks/month, 2 - 3 drinks/week, or $\geq 4$ drinks/week).

All participants underwent hepatic MRE using the technique as previously described $[17,18]$ with the following parameters: MR scanner $=1.5 \mathrm{~T}$ (GE, Wisconsin, USA); sequence $=$ GREMRE; mechanical frequency $=$ $60 \mathrm{~Hz}$; phase offsets $=4$; Motion encoding sensitivity $(\mathrm{MENC})=32 \mu \mathrm{m} /($ pi radian $)$; imaging plane = axial; motion sensitizing direction $=$ axial; Field of view $(\mathrm{FOV})$ $=32-44 \mathrm{~cm}$; matrix $=256 \times 96$; fractional phase $\mathrm{FOV}=$ $0.75-1$; flip angle $=30^{\circ}$; Number of excitation $(\mathrm{NEX})=$ 1; Bandwidth $=31.25 \mathrm{kHz}$; TE $/ \mathrm{TR}=24.5 / 50 \mathrm{msec}$; slice thickness $=10 \mathrm{~mm}$; number of slices $=2$; scan time $=4$ breath-holds $(4 \times 17 \mathrm{sec})$. Mean liver stiffness values were reported in the region of interest (ROI) drawn on the liver region of the elastogram where wave SNR was high, avoiding large blood vessels found in the MRE magnitude images and severe wave interferences found in wave images. Prior analyses have shown that a diagnostic cut-off value of 2.9 kilopascals $(\mathrm{kPa})$ can predict 
all stages of liver fibrosis when compared to normal liver with a sensitivity and specificity of $98 \%$ and $99 \%$, respectively [18]. Those with an abnormal scan $(>2.9 \mathrm{kPa})$ were offered a consultation in the hepatobiliary clinic and the decision for a liver biopsy was based on clinical judgment. This included taking into account the total MTX dose, risk factors for NASH, and abnormal laboratory values (AST, albumin).

\subsection{Statistical Analysis}

Patient characteristics were summarized as mean and standard deviation or number and percentage, as appropriate. Logistic regression and linear regression models with and without adjustment were used to assess the association between mean liver stiffness value by MRE and variables of interest. Results of logistic regression models were presented as odds ratios with $95 \%$ confidence intervals. The confidence intervals were estimated assuming the log odds ratio was approximately normally distributed. Statistical analysis was performed using SAS, version 9 (SAS Institute, Inc., Cary, NC).

\section{Results}

A total of 65 patients underwent hepatic MRE scanning. MRE scan examples are shown in Figure 1 (normal) and Figure 2 (abnormal). Patient demographic and clinical data are contained in Table $\mathbf{1}$.

Neither the total cumulative MTX dose nor the duration of MTX treatment was associated with mean liver stiffness value in unadjusted linear regression $(p=0.51$ and $\mathrm{P}=0.20$, respectively).

The continuous mean liver stiffness value (in $\mathrm{kPa}$ ) was significantly associated with BMI $(\mathrm{p}=0.015, \mathrm{r}$-square $=$ $0.09)$ on linear regression. Using logistic regression analyses, having a mean liver stiffness value $>2.9 \mathrm{kPa}$ was associated with increased BMI (OR $=1.18$ per $1 \mathrm{~kg} / \mathrm{m}^{2}$; $95 \%$ CI: $1.03,1.36 ; \mathrm{p}=0.017$ ). A plot of liver stiffness by MRE vs. body mass index is provided in Figure 3. BMI remained significantly associated with mean liver stiffness value following adjustment for MTX dose or MTX duration. Mean liver stiffness value was not significantly associated with age, sex, or duration of RA.

The association between elevated mean liver stiffness value and BMI remained significant (OR: $1.24 ; 95 \% \mathrm{CI}$ : 1.04, 1.48; $\mathrm{p}=0.019$ ) after adjustment for DM, IFG and hyperlipidemia. Only 2 patients had DM. Patients with DM were somewhat more likely to have higher mean liver stiffness values, but this association was not significant (OR: 9.5; $\mathrm{p}=0.13$ ). Twelve patients had IFG (including the 2 DM patients). IFG was not associated with abnormal mean liver stiffness values $(p=0.37)$. Twenty-one patients had hyperlipidemia, patients with hyperlipidemia were somewhat more likely to have ab-



(a)

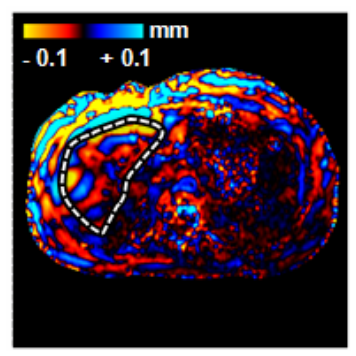

(b)



(c)

Figure 1. An example of normal MRE scan. (a) Anatomic image; (b) MRE wave image; (c) MR elastogram. Liver stiffness $($ Mean \pm SD) $=2.07 \pm 0.30 \mathrm{kPa}$. This patient was 70 year old, female; height $=1.63 \mathrm{~m}$, weight $=64 \mathrm{~kg}$, $\mathrm{BMI}=$ $24.1 \mathrm{~kg} / \mathrm{m}^{2}$. RA was diagnosed in 1970 . At the time of MRE scan, the patient was on MTX for almost 24 years. The total dose of MTX was $18,468 \mathrm{mg}$.

normal mean liver stiffness values, but this association was not significant (OR: 1.7; $\mathrm{p}=0.53$ ). Eleven patients reported using alcohol more than once a month. Alcohol use was not associated with abnormal mean liver stiffness values $(p=0.96)$.

Forty-five patients had at least one abnormal AST value during treatment with MTX. There was no association between having ever had any abnormal AST during MTX treatment and abnormal mean liver stiffness values $(p=0.39)$, however, there was a significant association between elevated mean liver stiffness and frequency of abnormal AST values for the duration of methotrexate treatment and in the preceding 2 years prior to the scan ( $\mathrm{p}$ $=0.035$ and $p=0.31$, respectively). Characteristics of the patients with an abnormal mean liver stiffness values and results of subsequent liver biopsy are contained in Table 2. 


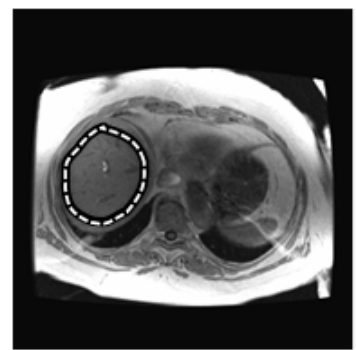

(a)

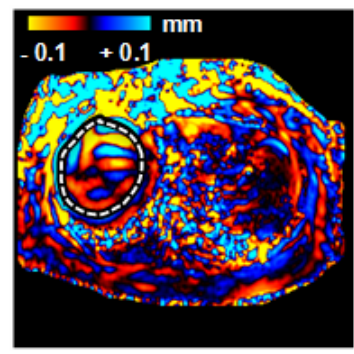

(b)



(c)

Figure 2. An example of abnormal MRE scan (patient \#2). (a) Anatomic image; (b) MRE wave image; (c) MR elastogram. Liver stiffness $($ Mean \pm SD) $=3.46 \pm 0.59 \mathrm{kPa}$. This patient was 65 year old, female; height $=1.57 \mathrm{~m}$, weight $=$ $94 \mathrm{~kg}, \mathrm{BMI}=37.9 \mathrm{~kg} / \mathrm{m}^{2}$. RA was diagnosed in 1985 . At the time of MRE scan, the patient was on MTX for 3.3 years. The total dose of MTX was $2950 \mathrm{mg}$. Liver biopsy was performed after MRE scan: Mild reactive hepatitis with mild portal fibrosis (stage 1 of 4).

All but 1 of the 7 patients with an abnormal mean liver stiffness had a BMI $>30 \mathrm{~kg} / \mathrm{m}^{2}$, and of those with high BMI, patients \#1, \#4, and \#7 had several abnormal AST values in the 2 years prior to the scan. Five of these 7 patients subsequently underwent liver biopsy. Histopathological examination revealed evidence of steatohepatitis in all 5, and all but 1 had evidence for fibrosis. Due to the severity of findings on liver biopsy, patient \#4 discontinued MTX. The remaining patients with an abnormal liver biopsy remained on MTX as it was determined that findings on liver biopsy were not clinically significant. It was recommended that these patients modify risk factors for NASH, continue laboratory monitoring of liver tests, and to undergo repeat MRE within the next few years to reduce the likelihood of liver disease progression.
Table 1. Demographic and clinical features of 65 patients with rheumatoid arthritis receiving methotrexate who underwent hepatic magnetic resonance elastography (MRE) scanning.

\begin{tabular}{lll}
\hline \multicolumn{1}{c}{ Variable } & N & \multicolumn{1}{c}{$\begin{array}{c}\text { N (\%) or } \\
\text { mean } \pm \text { s.d. }\end{array}$} \\
\hline Age (years) & 65 & $61.7 \pm 11.0$ \\
Female sex & 65 & $50(77 \%)$ \\
Duration RA (years) & 65 & $15.2 \pm 10.7$ \\
BMI (kg/m ${ }^{2}$ ) & 65 & $30.1 \pm 6.5$ \\
RF positivity & 63 & $46(73 \%)$ \\
CCP antibody positivity & 37 & $15(41 \%)$ \\
Cumulative MTX dose (mg) & 65 & $6551.1 \pm 3493.5$ \\
MTX treatment duration (months) & 65 & $112.1 \pm 59.9$ \\
Folate supplementation & 65 & $64(98 \%)$ \\
Mean liver stiffness (kPa) & 65 & $2.30 \pm 0.43$ \\
AST value ever abnormal & 63 & $45(71 \%)$ \\
$>50 \%$ of AST values abnormal ever & 65 & $4(6 \%)$ \\
$>50 \%$ of AST values abnormal 2007-2008 & 65 & $1(2 \%)$ \\
Hyperlipidemia & 65 & $21(32 \%)$ \\
Diabetes mellitus & 65 & $2(3 \%)$ \\
Impaired fasting blood glucose & 62 & $12(19 \%)$ \\
Alcohol use $>1$ X/month & 60 & $11(18 \%)$ \\
\hline
\end{tabular}

"2007-2008 corresponds to 2 years prior to MRE scan. Abbreviations: AST = aspartate aminotransferase; BMI = body mass index; MTX = methotrexate; $\mathrm{kPa}=$ kilopascal; $\mathrm{RA}=$ rheumatoid arthritis; $\mathrm{RF}=$ rheumatoid factor; $\mathrm{CCP}=$ anti-citrullinated protein antibody.



Figure 3. Plot of liver stiffness by magnetic resonance elastography vs body mass index. Solid line shows the trend with smoothing. Dashed line shows the cutpoint for abnormal liver stiffness (2.9 kPa).

Patient \#5 had an abnormal AST of up to twice normal at the time of the MRE scan. This patient was seen in hepatology consultation shortly thereafter; repeat AST at that point was normal. Therefore, in view of lack of risk factors for NASH or other chronic liver conditions and the decision to discontinue MTX due to aphthous ulcers, this patient did not undergo a liver biopsy. Rather, a re- 
Table 2. Abnormal hepatic magnetic resonance elastography (MRE) scans in patients receiving methotrexate for treatment of rheumatoid arthritis.

\begin{tabular}{|c|c|c|c|c|c|c|c|}
\hline Patient & $\begin{array}{c}\text { Age } \\
\text { (years) }\end{array}$ & Sex & $\begin{array}{c}\mathrm{BMI} \\
\left(\mathrm{kg} / \mathrm{m}^{2}\right)\end{array}$ & $\begin{array}{l}\text { Total MTX } \\
\text { dose (mg) }\end{array}$ & $\begin{array}{l}\text { Frequency abnormal AST } \\
2 \text { years prior to MRE }\end{array}$ & $\begin{array}{c}\text { Mean liver } \\
\text { stiffness value }(\mathrm{kPa})\end{array}$ & Liver biopsy findings \\
\hline 1 & 71 & $\mathrm{~F}$ & 31.1 & 14,235 & $4 / 9$ & 3.36 & $\begin{array}{l}\text { Mildly active (grade } 1 \text { of } 3 \text { ) steatohepatitis with focal. } \\
\text { pericellular fibrosis and mild portal fibrosis (stage } 1 \text { of } 4 \text { ). }\end{array}$ \\
\hline 2 & 65 & $\mathrm{~F}$ & 37.9 & 2950 & $1 / 13$ & 3.46 & $\begin{array}{l}\text { Mild reactive hepatitis with mild portal fibrosis (stage } 1 \\
\text { of } 4 \text { ). }\end{array}$ \\
\hline 3 & 57 & $\mathrm{~F}$ & 42.1 & 11,880 & $0 / 15$ & 3.23 & Mildly active steatohepatitis without fibrosis. \\
\hline 4 & 46 & $\mathrm{~F}$ & 44.9 & 6875 & $5 / 7$ & 2.92 & $\begin{array}{l}\text { Moderately active steatohepatitis, grade } 2 \text { (of } 3 \text { ) with } \\
\text { portal, periportal, and pericellular fibrosis, stage } 2 \text { (of } 4 \text { ). }\end{array}$ \\
\hline 5 & 65 & $\mathrm{~F}$ & 27.4 & 6340 & $1 / 8$ & 3.26 & $\begin{array}{l}\text { Not pursued; repeat MRE showed results within a normal } \\
\text { range. }\end{array}$ \\
\hline 6 & 66 & M & 37.3 & 3680 & $0 / 10$ & 3.13 & Mild fatty change with mild portal fibrosis. \\
\hline 7 & 62 & $\mathrm{~F}$ & 31.8 & 9295 & $7 / 17$ & 3.15 & Discontinued MTX; no biopsy performed. \\
\hline
\end{tabular}

Abbreviations: $\mathrm{AST}=$ aspartate aminotransferase; $\mathrm{BMI}=$ body mass index; $\mathrm{MTX}=$ methotrexate; $\mathrm{kPa}=$ kilopascal.

peat MRE scan was performed approximately 4 months after the initial scan, and this revealed normalization of the mean liver stiffness value.

Of the 7 patients with abnormal mean liver stiffness values, none reported drinking greater than 1 alcohol containing drink per month.

\section{Discussion}

Findings of our cross sectional study indicate that neither MTX cumulative dose nor treatment duration correlated with mean liver stiffness values. BMI is associated with hepatic inflammation and fibrosis as assessed by MRE, independent of MTX cumulative dose or treatment duration. The finding of an abnormal AST value at some point while on MTX for RA is not uncommon, and is not associated with hepatic inflammation in our study. However, the frequency of AST abnormalities ever and in the preceding 2 years prior to the scan were associated with elevated mean liver stiffness values.

There have been inconsistent findings reported in the literature regarding whether total cumulative dose of MTX is associated with the presence of fibrosis in patients receiving this drug for the treatment of RA [12]. Our findings on MRE in this study did not support such an association. In agreement with our findings, a recent study performing transient elastography for the non-invasive assessment of liver fibrosis in RA patients and did not detect an association between abnormal scans and total cumulative dose of MTX [19]. Such conflicting data may be due to lack of full consideration of confounding factors, including those associated with NASH.

Much of the histologic data for liver fibrosis in RA patients receiving MTX comes from studies prior to the development of MTX laboratory monitoring guidelines in 1994, when routine biopsies were performed for assessment of liver damage. Although many studies have not taken into account confounding factors for liver disease, some risk factors for liver fibrosis identified have included excessive alcohol intake, older age, and diabetes mellitus $[8,9,20,21]$. Consistent with our findings, obesity has been reported as a risk factor by others $[8,9,22]$.

Obesity is a major underlying determinant of non-alcoholic fatty liver disease (NAFLD). The prevalence of NAFLD is estimated to be $30 \%$ [23], and may be increasing with the current obesity epidemic. Although NAFLD a benign entity, up to one-quarter may progress to NASH, which has important clinical implications, as it can lead to end stage liver disease [24].

Some risk factors for MTX toxicity parallel those of NAFLD and NASH, such as diabetes mellitus and obesity [25]. It may also be that liver test abnormalities are detected more frequently in RA patients receiving MTX who have known NASH because these patients may undergo more frequent liver test monitoring out of heightened clinician concern for hepatotoxicity in these patients [4]. Some authors have questioned whether the apparent increase in hepatic toxicity with MTX use in patients with psoriasis is a result of NASH, as there seems to be a higher prevalence of metabolic syndrome in these patients [26].

The association of elevated BMI and abnormal mean liver stiffness values independent of MTX dose or duration suggests that NASH likely is in part responsible for hepatic inflammation and/or fibrosis found in the RA patients in this study. The degree to which MTX contributes to hepatic damage is unclear. Histologic findings attributed to MTX are non-specific and resemble those of NASH [25].

Abnormal liver tests are not uncommon in patients re- 
ceiving MTX, consistent with our findings, in which $45 / 65(71 \%)$ of patients had an abnormal value during their treatment course. An abnormal AST while receiving MTX at some point was not associated with an elevated mean liver stiffness value in our study. There was, however, a significant association for frequency of abnormal AST values during MTX treatment and in the preceding 2 years prior to the scan and an elevated mean liver stiffness value.

Current guidelines suggest further evaluation including liver biopsy based on the frequency of abnormal AST values during one year (5/9) [2]. In our study, of the four patients with an abnormal mean liver stiffness value and biopsy findings of hepatic inflammation and fibrosis, only two were monitored every 2 months, on average, and the other two, less frequently. Whether those who were monitored less frequently would have ultimately had $\geq 50 \%$ abnormal AST per year is uncertain, though the two patients monitored regularly had few abnormal AST values. Liver fibrosis would not have been suspected or found without the MRE scan in these patients. The finding of fibrosis by MRE lead to a change in clinical management in some patients: in one, MTX was discontinued, and in the others, risk factor modification, particularly in the form of weight loss, was advised on the basis of the high likelihood of NASH in these patients.

This is the first study to use MRE for assessment of liver fibrosis patients in RA patients receiving MTX. Strengths of the study include the use of this reliable non-invasive methodology which has the advantage of assessing the entire liver to provide an estimate of likelihood of disease, in contrast to the more limited assessment possible on liver biopsy or ultrasonographic transient elastography. Unlike transient elastography, MRE is not influenced by BMI, ascites or body habitus $[15,18]$. Though there was a wide range, the average MTX treatment duration in this study group was 9.3 years, which is longer than the less than 5 years of treatment in patients who constituted the cohort upon which much of the histologic data for monitoring guidelines was obtained.

Limitations of this study included its cross-sectional design limiting the ability to make causal inferences. Second, our sample size was relatively small, with relatively few abnormal tests, limiting its statistical power. Third, clinical variables were obtained through retrospective review of the clinical record. Fourth, though the standard laboratory monitoring for patients on MTX includes obtaining an AST value every 6 - 8 weeks, some patients may have had more or less frequent monitoring.

\section{Conclusion}

The results of our study suggest that patients with RA receiving MTX who have elevated BMI are at increased for hepatic inflammation, regardless of MTX cumulative dose. This may be ascribed to NASH. Hepatic MRE may provide a reliable, non-invasive assessment of liver fibrosis in patients with RA receiving MTX who are at risk for NASH. Further investigation in a greater number of obese RA patients with MRE may clarify these findings. Investigation of the utility of MRE in patients with psoriasis or psoriatic arthritis receiving MTX may also be particularly fruitful, as present dermatology guidelines still suggest routine liver biopsies in these patients [27].

\section{Future Perspective}

Noninvasive monitoring for liver fibrosis with hepatic MRE will likely emerge as a robust imaging method. It has the capability to enhance the detection of liver damage in a variety of different clinical scenarios including those related to concerns regarding methotrexate induced liver toxicity.

\section{Disclosure}

Dr. Richard L. Ehman, a co-author, has a potential financial interest associated with technology used in this project. That technology involves Magnetic Resonance Elastography. This technology was licensed to a third party via a fully paid-up, non-exclusive license in 2003.

\section{Authors Contributions}

DH participated in the design and coordination of the study, interpretation of data, and drafted the manuscript. JC also participated in the design and coordination of the study, performed as well as analyzed the MRE data and also contributed to drafting of the manuscript. RE helped to design the study and assisted with analysis of MRE data. JT and CM contributed to the design of the study and JT assisted with the interpretation of data. MY contributed with the development of hepatic MRE techniques. CC assisted with statistical analysis. EM helped with design, coordination of the study, and interpretation of data. All authors read and approved the final manuscript.

\section{Acknowledgements and Funding}

We wish to thank Jennifer Sletten, Heidi Hanf, Jane Jaquith, and Jeaneen Alcorn for their assistance as study coordinators. We also would like to thank Dr. Kevin Glaser for his assistance with hepatic MRE techniques.

Partially supported by NIH Grant EB001984.

\section{REFERENCES}

[1] T. R. Mikuls and J. O'Dell, "The Changing Face of Rheumatoid Arthritis Therapy: Results of Serial Surveys," Ar- 
thritis and Rheumatism, Vol. 43, No. 2, 2000, pp. 464465.

doi:10.1002/1529-0131(200002)43:2<464::AID-ANR30> 3.0.CO;2-U

[2] J. M. Kremer, G. S. Alarcon, R. W Lightfoot Jr., R. F. Wilkins, D. E. Furst, H. J. Williams, et. al., "Methotrexate for Rheumatoid Arthritis. Suggested Guidelines for Monitoring Liver Toxicity. American College of Rheumatology," Arthritis and Rheumatism, Vol. 37, No. 3, 1994, pp. 316-328. doi:10.1002/art.1780370304

[3] J. M. Kremer, D. E. Furst, M. E. Weinblatt and S. D. Blotner, "Significant Changes in Serum AST Across Hepatic Histological Biopsy Grades: Prospective Analysis of 3 Cohorts Receiving Methotrexate Therapy for Rheumatoid Arthritis," Journal of Rheumatology, Vol. 23, No. 3, 1996, pp. 459-461.

[4] P. D. Kent, H. S. Luthra and C. Michet Jr., "Risk Factors for Methotrexate-Induced Abnormal Laboratory Monitoring Results in Patients with Rheumatoid Arthritis," Journal of Rheumatology, Vol. 31, No. 9, 2004, pp. 17271731.

[5] Y. Yazici, D. Erkan, M. J. Harrison, N. P. Nikolov and S. A. Paget, "Methotrexate Use in Rheumatoid Arthritis Is Associated with Few Clinically Significant Liver Function Test Abnormalities," Clinical and Experimental Rheumatology, Vol. 23, No. 4, 2005, pp. 517-520.

[6] J. M. Kremer, "Not Yet Time to Change the Guidelines for Monitoring Methotrexate Liver Toxicity: They Have Served Us Well," Journal of Rheumatology, Vol. 29, No. 8, 2002, pp. 1590-1592.

[7] P. Angulo, "Nonalcoholic Fatty Liver Disease," New England Journal of Medicine, Vol. 346, No. 16, 2002, pp. 1221-1231. doi:10.1056/NEJMra011775

[8] J. M. Kremer, R. G. Lee and K. G. Tolman, "Liver Histology in Rheumatoid Arthritis Patients Receiving LongTerm Methotrexate Therapy. A Prospective Study with Baseline and Sequential Biopsy Samples," Arthritis and Rheumatism, Vol. 32, No. 2, 1989, pp. 121-127. doi:10.1002/anr.1780320202

[9] C. A. Phillips, P. J. Cera, T. F. Mangan and E. D. Newman, "Clinical Liver Disease in Patients with Rheumatoid Arthritis Taking Methotrexate," Journal of Rheumatology, Vol. 19, No. 2, 1992, pp. 229-233.

[10] P. D. Hall, M. J. Ahern, L. R. Jarvis, P. Stoll, M. A. Jenner and H. Harley, "Two Methods of Assessment of Methotrexate Hepatotoxicity in Patients with Rheumatoid Arthritis," Annals of the Rheumatic Diseases, Vol. 50, No. 7, 1991, pp. 471-476. doi:10.1136/ard.50.7.471

[11] R. F. Willkens, P. A. Leonard, D. O. Clegg, K. G. Tolman, J. R. Ward, C. R. Marks, M. L. Greene, G. J. Roth, C. G. Jackson, G. W. Cannon, et. al., "Liver Histology in Patients Receiving Low Dose Pulse Methotrexate for the Treatment of Rheumatoid Arthritis," Annals of the Rheumatic Diseases, Vol. 49, No. 8, 1990, pp. 591-593. doi:10.1136/ard.49.8.591

[12] S. G. West, "Methotrexate Hepatotoxicity," Rheumatic Diseases Clinics of North America, Vol. 23, No. 4, 1997, pp. 883-915. doi:10.1016/S0889-857X(05)70365-3
[13] Y. Yazici, T. Sokka, H. Kautiainen, C. Swearingen, I. Kulman and T. Pincus, "Long Term Safety of Methotrexate in Routine Clinical Care: Discontinuation Is Unusual and Rarely the Result of Laboratory Abnormalities," Annals of the Rheumatic Diseases, Vol. 64, No. 2, 2005, pp. 207-211. doi:10.1136/ard.2004.023408

[14] P. Asbach, D. Klatt, U. Hamhaber, J. Braun, R. Somasundaram, B. Hamm and I. Sack, "Assessment of Liver Viscoelasticity Using Multifrequency MR Elastography," Magnetic Resonance Medicine, Vol. 60, No. 2, 2008, pp. 373-379. doi: $10.1002 / \mathrm{mrm} .21636$

[15] L. Huwart, C. Sempoux, E. Vicaut, N. Salameh, L. Annet, E. Danse, F. Peeters, L. C. ter Beek, J. Rahier, R. Sinkus, Y. Horsmans and B. E. Van Beers, "Magnetic Resonance elastography for the Noninvasive Staging of Liver Fibrosis," Gastroenterology, Vol. 135, No. 1, 2008, pp. 32-40. doi:10.1053/j.gastro.2008.03.076

[16] O. Rouviere, M. Yin, M. A. Dresner, L. J. Burgart, J. L. Fidler and R. L. Ehman, "MR Elastography of the Liver: Preliminary Results," Radiology, Vol. 240, No. 2, 2006, pp. 440-448. doi:10.1148/radiol.2402050606

[17] J. A. Talwalkar, "Elastography for Detecting Hepatic Fibrosis: Options and Considerations," Gastroenterology, Vol. 135, No. 1, 2008, pp. 299-302. doi:10.1053/j.gastro.2008.05.038

[18] M. Yin, J. A. Talwalkar, K. J. Glaser, A. Manduca, R. C. Grimm, P. J. Rossman, J. L. Fidler and R. L. Ehman, "Assessment of Hepatic Fibrosis with Magnetic Resonance Elastography," Clinics in Gastroenterology and Hepatology, Vol. 5, No. 10, 2007, pp. 1207-1213.

[19] S. H. Park, J. Y. Choe and S. K. Kim, "Assessment of Liver Fibrosis by Transient Elastography in Rheumatoid Arthritis Patients Treated with Methotrexate," Joint Bone Spine, Vol. 77, No. 6, 2010, pp. 588-592. doi:10.1016/j.jbspin.2010.02.024

[20] A. M. Walker, D. Funch, N. A. Dreyer, K. G. Tolman, J. M. Kremer, G. S. Alarcon, R. G. Lee and M. E. Weinblatt, "Determinants of Serious Liver Disease among Patients Receiving Low-Dose Methotrexate for Rheumatoid Arthritis," Arthritis and Rheumatism, Vol. 36, No. 3, 1993, pp. 329-335. doi:10.1002/art.1780360307

[21] Q. E. Whiting-O'Keefe, K. H. Fye and K. D. Sack, "Methotrexate and Histologic Hepatic Abnormalities: A Meta-Analysis," American Journal of Medicine, Vol. 90, No. 6, 1991, pp. 711-716. doi:10.1016/S0002-9343(05)80060-9

[22] W. J. Shergy, R. P. Polisson, D. S. Caldwell, J. R. Rice, D. S. Pisetsky and N. B. Allen, "Methotrexate-Associated Hepatotoxicity: Retrospective Analysis of 210 Patients with Rheumatoid Arthritis," American Journal of Medicine, Vol. 85, No. 6, 1988, pp. 771-774. doi:10.1016/S0002-9343(88)80019-6

[23] B. A. Neuschwander-Tetri and S. H. Caldwell, "Nonalcoholic Steatohepatitis: Summary of an AASLD Single Topic Conference," Hepatology, Vol. 37, No. 5, 2003, pp. 1202-1219. doi:10.1053/jhep.2003.50193

[24] J. Jou, S. S. Choi and A. M. Diehl, "Mechanisms of Disease Progression in Nonalcoholic Fatty Liver Disease," Seminars in Liver Disease, Vol. 28, No. 4, 2008, pp. 370- 
379. doi:10.1055/s-0028-1091981

[25] G. Langman, P. M. Hall and G. Todd, "Role of NonAlcoholic Steatohepatitis in Methotrexate-Induced Liver Injury," Journal of Gastroenterology and Hepatology, Vol. 16, No. 12, 2001, pp. 1395-1401. doi:10.1046/j.1440-1746.2001.02644.x

[26] R. S. Azfar and J. M. Gelfand, "Psoriasis and Metabolic Disease: Epidemiology and Pathophysiology," Current Opin-

\section{List of Abbreviations}

MRE = Magnetic resonance elastography;

$\mathrm{RA}=$ Rheumatoid arthritis;

MTX = Methotrexate;

$\mathrm{kPa}=$ Kilopascals; ion in Rheumatology, Vol. 20, No. 4, 2008, pp. 416-422. doi:10.1097/BOR.0b013e3283031c99

[27] H. H. Roenigk Jr., R. Auerbach, H. Maibach, G. Weinstein and M. Lebwohl, "Methotrexate in Psoriasis: Consensus Conference," Journal of the American Academy of Dermatology, Vol. 38, No. 3, 1998, pp. 478-485. doi:10.1016/S0190-9622(98)70508-0
$\mathrm{AST}=$ Aspartate transaminase;

$\mathrm{BMI}=$ Body mass index;

$\mathrm{NASH}=$ Nonalcoholic steatohepatitis;

$\mathrm{DM}=$ Diabetes mellitus;

IFG $=$ Impaired fasting glucose . 